\title{
Stability and spatial coherence of nonresonantly pumped exciton-polariton condensates
}

\author{
Nataliya Bobrovska, ${ }^{1}$ Elena A. Ostrovskaya, ${ }^{2}$ and Michał Matuszewski ${ }^{1}$ \\ ${ }^{1}$ Institute of Physics Polish Academy of Sciences, Aleja Lotników 32/46, 02-668 Warsaw, Poland \\ ${ }^{2}$ Nonlinear Physics Centre, Research School of Physical Sciences and Engineering, The Australian National University, \\ Canberra ACT 0200, Australia
}

(Received 13 August 2014; revised manuscript received 27 October 2014; published 17 November 2014)

\begin{abstract}
We investigate the stability and coherence properties of one-dimensional exciton-polariton condensates under nonresonant pumping. We model the condensate dynamics using the open-dissipative Gross-Pitaevskii equation. In the case of spatially homogeneous pumping, we find that the instability of the steady state leads to significant reduction of the coherence length. We consider two effects that can lead to the stabilization of the steady state, i.e., the polariton energy relaxation and the influence of an inhomogeneous pumping profile. We find that, while the former has little effect on the stability, the latter is very effective in stabilizing the condensate, which results in a large coherence length.
\end{abstract}

DOI: 10.1103/PhysRevB.90.205304

PACS number(s): 67.85.De, 71.36.+c, 03.75.Kk

\section{INTRODUCTION}

Exciton polaritons are light-matter bosonic quasiparticles created due to strong coupling between excitons and photons [1-3]. Their extremely light effective mass combined with strong exciton-mediated interparticle interactions makes them an ideal system for the investigation of fundamental phenomena such as room-temperature Bose-Einstein condensation [4-6] as well as applications [7-11]. A wide range of phenomena observed in exciton-polariton systems, such as superfluidity [12], or spontaneous creation of self-localized structures, including solitons and vortices, has attracted great interest in recent years $[13,14]$. In contrast to condensates of ultracold atoms, polariton superfluids are nonequilibrium systems in which continuous pumping is required to maintain the condensate population $[1,3,14,15]$.

In a number of experiments, spatial coherence extending over the whole polariton cloud was demonstrated $[3,14,16-18]$. Recently, stability limits for polariton condensates under nonresonant pumping were determined within the open-dissipative Gross-Pitaevskii model [19]. It was predicted that spatially homogeneous steady states are stable in regions of parameter space determined by the ratio $\gamma_{R} g_{C} / \gamma_{C} g_{R}$, where $\gamma_{C, R}^{-1}$ are the lifetimes of the polariton condensate and the exciton reservoir and $g_{C, R}$ are the coefficients of interaction within the condensate and between the condensate and the reservoir, respectively. Importantly, for comparable interaction constants, stability close to the threshold was predicted only for relatively small values of $\gamma_{C} / \gamma_{R}$. It is important to note that values of $\gamma_{R}$ varying by orders of magnitude are used throughout the literature [20-22]. According to independent measurements $[23,24]$, physical values of $\gamma_{R}$ correspond to the unstable regime, where, as we show below, a significant reduction of the coherence length can be expected.

In this work, we investigate whether large coherence length can emerge in condensates even in the unstable region of parameter space predicted by the homogeneous theory. We analyze the stability and coherence properties of polariton condensates in more detail, taking into account the effects of polariton relaxation and the inhomogeneous pumping profile. We use the Bogoliubov-de Gennes theory as well as direct numerical integration of the open-dissipative Gross-Pitaeskii equations. We demonstrate that full coherence can be achieved even for large values of $\gamma_{C} / \gamma_{R}$ once the finite size of the pumping spot is taken into account. We believe that further experiments, in particular with homogeneous or ring-shaped pumping profiles, are necessary to determine stability limits in parameter space and fix the values of phenomenological parameters of the model. In particular, the observation of coherence reduction or its absence in the case of almosthomogeneous pumping would verify whether the ratio $\gamma_{C} / \gamma_{R}$ corresponds to the unstable regime.

\section{THE MODEL}

In one dimension (e.g., in a microwire [17]), the excitonpolariton condensate with the wave function $\psi(x, t)$ can be modeled with the generalized open-dissipative GrossPitaevskii equation coupled to the rate equation for the polariton reservoir density, $n_{R}(x, t)[15,25]$,

$$
\begin{aligned}
i \hbar \frac{\partial \psi}{\partial t}= & -\frac{\hbar^{2}}{2 m^{*}} \frac{\partial^{2} \psi}{\partial x^{2}}+g_{C}^{1 \mathrm{D}}|\psi|^{2} \psi+g_{R}^{1 \mathrm{D}} n_{R} \psi \\
& +i \frac{\hbar}{2}\left(R^{1 \mathrm{D}} n_{R}-\gamma_{C}\right) \psi, \\
\frac{\partial n_{R}}{\partial t}= & P(x)-\left(\gamma_{R}+R^{1 \mathrm{D}}|\psi|^{2}\right) n_{R},
\end{aligned}
$$

where $P(x)$ is the exciton creation rate determined by the pumping profile, $m^{*}$ is the effective mass of lower polaritons, $\gamma_{C}$ and $\gamma_{R}$ are the polariton and exciton loss rates, and $\left(R^{1 \mathrm{D}}, g_{i}^{1 \mathrm{D}}\right)=\left(R^{2 \mathrm{D}}, g_{i}^{2 \mathrm{D}}\right) / \sqrt{2 \pi d^{2}}$ are the rates of stimulated scattering into the condensate and the interaction coefficients, rescaled in the one-dimensional case. Here, we assumed a Gaussian transverse profile of $|\psi|^{2}$ and $n_{R}$ of width $d$. In the case of a one-dimensional microwire [17], the profile width $d$ is of the order of the microwire thickness. We note that the exciton field corresponds to the "active" exciton population rather than the reservoir at high-energy levels [21]. While the latter may have much longer lifetime $\gamma^{-1}$, it is not subject to a considerable backaction from polaritons, such as stimulated scattering, which is relevant for the stability properties of the system. 
By rescaling time, space, wave-function amplitude, and material coefficients as $t=\tau \widetilde{t}, x=\xi \tilde{x}, \psi=(\xi \beta)^{-1 / 2} \widetilde{\psi}, n_{R}=$ $(\xi \beta)^{-1} \widetilde{n}_{R}, \quad R^{1 \mathrm{D}}=(\xi \beta / \tau) \widetilde{R}, \quad\left(g^{1 \mathrm{D}}, g_{R}^{\mathrm{1D}}\right)=(\hbar \xi \beta / \tau)\left(\widetilde{g}, \widetilde{g}_{R}\right)$, $\left(\gamma, \gamma_{R}\right)=\tau^{-1}\left(\widetilde{\gamma}, \widetilde{\gamma}_{R}\right)$, and $P(x)=(1 / \xi \beta \tau) \widetilde{P}(x)$, where $\xi=\sqrt{\hbar \tau / 2 m^{*}}$, while $\tau$ and $\beta$ are arbitrary scaling parameters, we can rewrite the above equation in the dimensionless form (from now on we omit the tildas for convenience):

$$
\begin{gathered}
i \frac{\partial \psi}{\partial t}=\left[-\frac{\partial^{2}}{\partial x^{2}}+\frac{i}{2}\left(R n_{R}-\gamma\right)+g|\psi|^{2}+g_{R} n_{R}\right] \psi, \\
\frac{\partial n_{R}}{\partial t}=P(x)-\left(\gamma_{R}+R|\psi|^{2}\right) n_{R} .
\end{gathered}
$$

In particular, we may choose the time scaling $\tau$ in such a way that $\gamma_{R}=1$ without loss of generality. The norms of both fields $N_{\psi}=\int|\psi|^{2} d x$ and $N_{R}=\int n_{R} d x$ are scaled by the factor of $\beta$.

\section{HOMOGENEOUS PUMPING}

We investigate the stability of a uniform condensate solution with the assumption of homogeneous pumping $(P=$ const $)$,

$$
\begin{aligned}
\psi(x, t) & =\psi_{0} e^{-i \mu_{0} t}, \\
n_{R}(x, t) & =n_{R}^{0},
\end{aligned}
$$

where $\mu_{0}$ is the chemical potential of the condensate. Substitution of the above equations into Eq. (2) gives the steady-state solutions. For $P<P_{\text {th }}=\gamma \gamma_{R} / R$, only the noncondensed solution $\psi_{0}=0$ exists with $n_{R}^{0}=P / \gamma_{R}$. Above threshold, the condensate density is $\left|\psi_{0}\right|^{2}=(P / \gamma)-\left(\gamma_{R} / R\right)$ with the chemical potential $\mu_{0}=g\left|\psi_{0}\right|^{2}+g_{R} n_{R}^{0}$ and the reservoir density $n_{R}^{0}=\gamma / R[15,19]$.

The stability of the condensed state depends on the system parameters. In Fig. 1, we show typical examples of dynamics of the condensate density in stable, weakly unstable, and strongly unstable cases. Periodic boundary conditions are imposed, which corresponds to a ring-shaped geometry of the microwire. The initial state is perturbed by a white noise, which mimics classical (e.g., thermal) density fluctuations in the initial polariton field. We checked that stability is independent of the amplitude of the noise provided that it is much smaller than the amplitude of the steady state.

It is important to note that while in all three cases the population of low-momentum states is much larger than that below threshold, there is no long-range order in the final states in Figs. 1(b) and 1(c), even in the mean-field limit. As shown in Fig. 1(d), the coherence length is of the order of the typical size of the structures visible on the figures. The reason for the reduced coherence is different than in the case of one-dimensional (1D) quasicondensates of ultracold atoms [26-28] since here we work in the mean-field limit, and the same instability is present also in the higher-dimensional versions of the model. The above modulational instability of the condensate was first reported in [15], where it was named the "hole-burning effect." The analytical condition for stability was derived in [19]

$$
\frac{P}{P_{\mathrm{th}}}>\frac{g_{R}}{g} \frac{\gamma}{\gamma_{R}}
$$
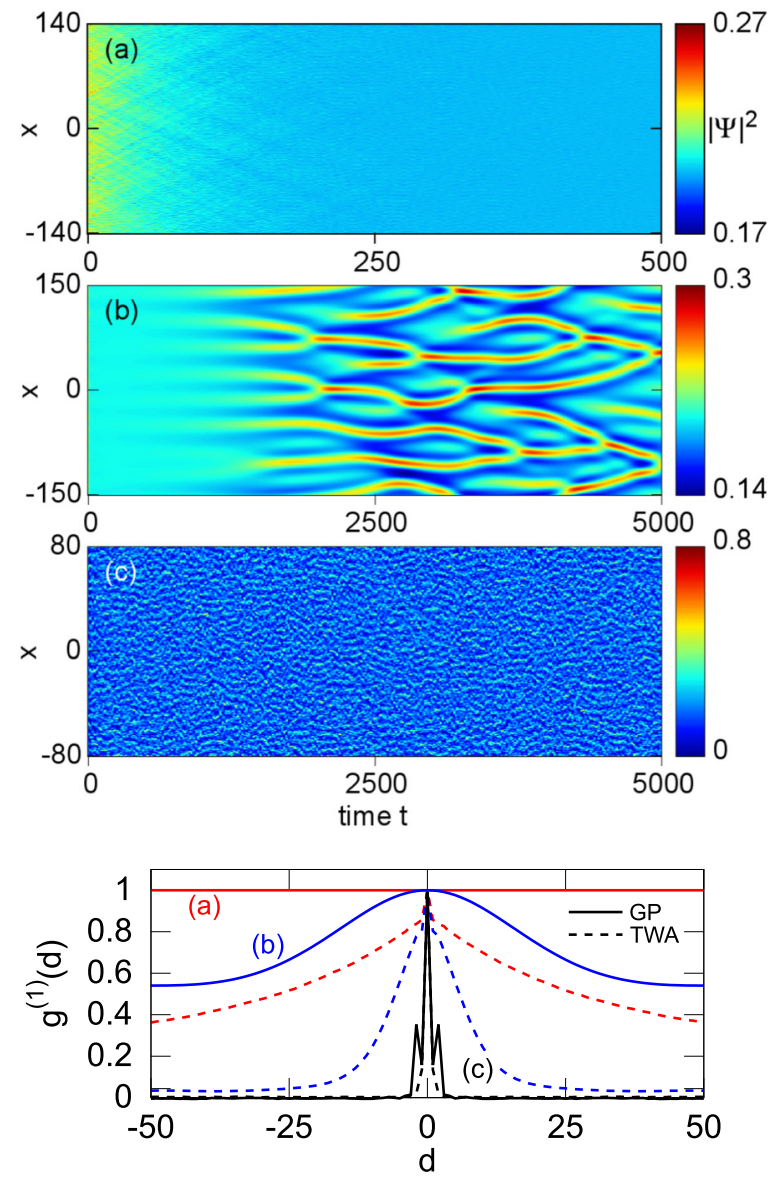

FIG. 1. (Color online) Evolution of the stationary state $\psi_{0}=\sqrt{\left(P-P_{\mathrm{th}}\right) / \gamma}$ with additional noise in the case of homogeneous pumping. The evolution of density in (a) the stable case and (b),(c) the unstable case near and far from the critical point, respectively. Parameters are $R=1, g=1, g_{R}=2 g$, $P / P_{\mathrm{th}}=1.2$, and $\gamma / \gamma_{R}=0.1,0.66,4.5$ for (a), (b), and (c), respectively. Bottom panel shows the first-order correlation functions $g^{(1)}(d)=\left\langle\psi^{*}(x) \psi(x+d)\right\rangle /\left\langle|\psi(x)|^{2}\right\rangle \quad$ at $\quad t=t_{\max }, \quad$ calculated from about 500 samples. Solid lines show $g^{(1)}$ calculated with Eqs. (1) and dashed lines depict the corresponding results with quantum fluctuations included (see text). Corresponding parameters in physical units are time unit $\tau=\gamma_{R}^{-1}=10 \mathrm{ps}$, length unit $\xi=3.4 \mu \mathrm{m}, g=3.4 \mu \mathrm{eV} \mu \mathrm{m}^{2}, \quad R=5.1 \times 10^{-3} \mu \mathrm{m}^{2} \mathrm{ps}^{-1}$ for $d=2 \mu \mathrm{m}, m^{*}=5 \times 10^{-5} m_{e}$, and $\beta=0.003$. TWA correlation lengths are (a) $\xi=70 \mu \mathrm{m}$, (b) $\xi=17 \mu \mathrm{m}$, and (c) $\xi=3 \mu \mathrm{m}$.

The stability limit for $g_{R}=2 g$ is marked in Fig. 2(a) with a thick black line.

The above result has important practical consequences. As shown in several experiments, the lifetime of polaritons is typically much shorter than the exciton lifetime. This can be easily understood since the cavity photon lifetime even in very high- $Q$ cavities [29] is still shorter than the natural lifetime of excitons, which are of the order of hundreds of picoseconds. The reported exciton lifetimes were as high as $\gamma_{R}^{-1}=700$ ps [23,24] or $\gamma_{R}^{-1}=300$ ps [20]. Taking into account that typical polariton lifetimes $\gamma^{-1}$ are of the order of a few or a few tens of picoseconds, these values correspond to values of $\gamma / \gamma_{R}$ in the phase diagram in Fig. 2(a), where stability can be achieved only at a very high pumping powers. 

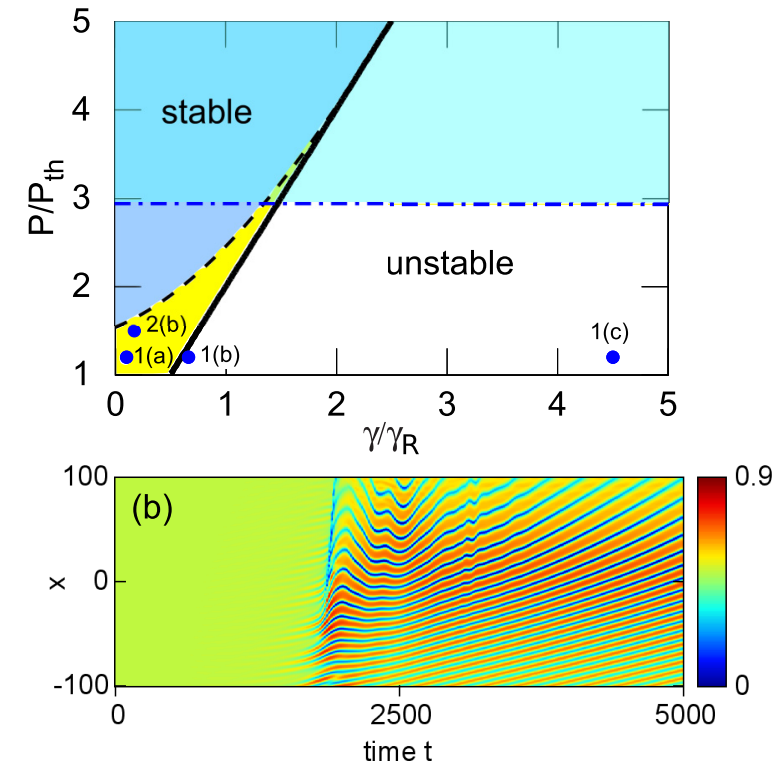

FIG. 2. (Color online) (a) Stability limits of a static condensate created with homogeneous pumping (solid line) and for a uniform condensate flowing with momentum $p=1$ (dashed line). Parameters are $R=1, g=1, g_{R}=2 g$, and $\gamma_{R}=1$. The dash-dotted line corresponds to the critical velocity condition $p=c_{s}=\sqrt{2 g|\psi|^{2}}$. The black solid line corresponds to the analytical condition (5) for stability of the homogeneous solution. Blue points correspond to particular cases from Fig. 1. (b) Evolution of $|\psi(x, t)|^{2}$ for a uniform flow with homogeneous pumping and momentum $p=1$ in the motionally unstable regime, $P / P_{\text {th }}=1.5, \gamma / \gamma_{R}=0.17$.

Here, we suggest how this fact can be reconciled with the emergence of large coherence length in experiments. It was pointed out that the lifetime of active excitons, i.e., excitons that can scatter directly to the condensate, can be much shorter than the average lifetime of excitons in the system [21]. Since these excitons become dressed with light field at low momenta, their lifetime can be reduced due to a nonzero photonic Hopfield coefficient. However, there is no fundamental reason why the lifetime should become shorter than the photon lifetime, which is necessary for stability at any power $P>P_{\text {th }}$ in Eq. (5). On the other hand, we show that this condition can be relaxed in the case of a finite pumping spot. We show that in the case of inhomogeneous pumping profiles, large coherence lengths can emerge for relatively large values of $\gamma / \gamma_{R}$.

\section{A. Quantum fluctuations}

We note that while the above calculations take into account only the classical fluctuations in the initial polariton field, it is possible to include the effect of quantum fluctuations on the level of classical fields approximation [30-32]. In the truncated Wigner approximation (TWA), the quantum field is simulated by an ensemble of realizations of the Gross-Pitaevskii equations in the form similar to (2) and (3), with the addition of a stochastic term. The extended version of Eq. (2) reads $d \psi=(\ldots) d t+d W$, where $d W$ is a complex stochastic variable with [30-32]

$$
\begin{aligned}
\left\langle d W(x) d W\left(x^{\prime}\right)\right\rangle & =0, \\
\left\langle d W(x) d W^{*}\left(x^{\prime}\right)\right\rangle & =\beta \frac{d t}{2 \Delta x}\left(R n_{R}+\gamma_{C}\right) \delta_{x, x^{\prime}},
\end{aligned}
$$

reflecting the quantum noise due to the particles entering and leaving the condensate. In Fig. 1 (bottom panel, dashed lines), we show the effect of quantum fluctuations on the correlation functions. While quantum fluctuations lead to decay of correlation functions over long distance [17,33,34], this effect is overwhelmed by the instability in the unstable case (c) (black lines), which marks a dramatic reduction of the correlation length.

\section{B. Stability of a uniform flow}

Another important issue in the case of uniform pumping is the stability of a condensate with a finite momentum. In this case, the stationary solution is

$$
\psi(x, t)=\psi_{0} e^{i p x-i \mu_{0} t},
$$

where $p$ is the condensate momentum and $\mu_{0}=g \psi_{0}^{2}+g_{R} n_{R}^{0}+p^{2}$ is the chemical potential. Such polariton flow can occur naturally, e.g., in the presence of a spatially varying exciton-photon detuning which generates a potential gradient, or due to repulsive interactions with reservoir excitons which are generated by the pump [17]. Similarly to the case of a static condensate $[15,19]$, the modulational stability of the steady-state solution (7) can be investigated within the Bogoliubov-de Gennes approximation [35,36]. Small fluctuations around the steady state have the form

$$
\begin{aligned}
\psi & =\psi_{0} e^{i p x-i \mu_{0} t}\left[1+\sum_{k}\left\{a_{k} e^{-i\left(\omega_{k} t-k x\right)}+b_{k}^{*} e^{i\left(\omega_{k}^{*} t-k x\right)}\right\}\right] \\
n_{R}(x, t) & =n_{R}^{0}\left[1+\sum_{k}\left\{c_{k} e^{-i\left(\omega_{k} t-k x\right)}+c_{k}^{*} e^{i\left(\omega_{k}^{*} t-k x\right)}\right\}\right]
\end{aligned}
$$

where $\omega_{k}$ is the frequency of the mode with the wave number $k$, and $a_{k}, b_{k}, c_{k}$ are small fluctuations. Substituting Eqs. (8) into the system of Eq. (2) and keeping linear terms only, we get the standard eigenvalue problem $\mathcal{L}_{k} \mathcal{U}_{k}=\omega_{k} \mathcal{U}_{k}$, where $\mathcal{U}_{k}=\left(a_{k}, b_{k}, c_{k}\right)^{T}$ and

$$
\mathcal{L}_{k}=\left(\begin{array}{ccc}
g \psi_{0}^{2}+2 k p+k^{2} & g \psi_{0}^{2} & \frac{i}{2} R+g_{R} \\
-g \psi_{0}^{2} & -g \psi_{0}^{2}+2 k p-k^{2} & \frac{i}{2} R-g_{R} \\
-i \gamma \psi_{0}^{2} & -i \gamma \psi_{0}^{2} & -i\left(\gamma_{R}+R \psi_{0}^{2}\right)
\end{array}\right)
$$

The existence of an eigenvalue $\omega_{k}$ with a positive imaginary part marks the dynamical instability of the flow.
We solved the above eigenvalue problem numerically to obtain the stability limits in parameter space. The results are 
presented in Fig. 2, where the stability of the flow with a finite momentum is marked with a dashed line. Similarly to [36], the region of stability of solutions is further decreased with respect to the static case. There is a certain area for small $P / P_{\text {th }}$ and $\gamma / \gamma_{R}$ in which the condensate becomes unstable. In this region, the dynamics of the instability is qualitatively different from the one presented in Fig. 1. Instead of chaotic dynamics, regular waves are created by the unstable flow, as shown in Fig. 2(b). Here, instead of periodic boundary conditions, we used boundary conditions that provide a flow of particles with a prescribed momentum through the borders of the computational window: $\partial \psi / \partial x=i p \psi$.

The origin of the above modulational instability of the flow is clearly due to the movement of polaritons with respect to the practically immobile reservoir excitons. This instability is absent in the standard version of the polariton model which does not include a separate equation for the reservoir [35,37], since in this case the system is Galilean invariant. However, it can be recovered by inclusion of frequency-dependent pumping [35], which resembles the momentum dependence of scattering from the immobile reservoir. Notably, similar to [35], the modulational instability does not correspond to $p$ being higher than the critical velocity; see dash-dotted line in Fig. 2(a). The latter condition defines the limit on stability of the flow with respect to scattering against defects.

\section{EFFECTS OF POLARITON ENERGY RELAXATION}

One of the drawbacks of the Gross-Pitaevskii model based on Eq. (2) is that the relaxation of polariton energy is not taken into account. Several experiments $[17,20,38]$ have shown that energy relaxation (or thermalization) may play an important role in the dynamics. It is important to investigate whether it can have a stabilizing effect on the condensate. To estimate the effect of relaxation, we follow Refs. [20,38] by adding a phenomenological relaxation term to the condensate evolution equation (2),

$$
\frac{\partial \psi}{\partial t}=\left[(i+A) \frac{\partial^{2}}{\partial x^{2}}+\frac{1}{2}\left(R n_{R}-\gamma\right)-i g|\psi|^{2}-i g_{R} n_{R}\right] \psi,
$$

where the real coefficient $A$ corresponds to the energy relaxation in the condensate. Following the steps of the previous section, we obtain the matrix which describes the Bogoliubov-de Gennes excitations in the static $(p=0)$ case,

$$
\mathcal{L}_{k}=\left(\begin{array}{ccc}
g \psi_{0}^{2}+i \alpha k^{2} & g \psi_{0}^{2} & \frac{i}{2} R+g_{R} \\
-g \psi_{0}^{2} & -g \psi_{0}^{2}+i \alpha k^{2} & \frac{i}{2} R-g_{R} \\
-i \gamma \psi_{0}^{2} & -i \gamma \psi_{0}^{2} & -i\left(\gamma_{R}+R \psi_{0}^{2}\right)
\end{array}\right),
$$

where $\alpha=i+A$.

The correction to the stability diagram with the relaxation term included is shown in Fig. 3, where we compare the stability limits obtained numerically by solving (11) with the analytical condition for the relaxation-free case (5). It is clear that even the large relaxation rate $(A=0.5)$ cannot lead to the stabilization of the condensate in a large parameter range, although the region of stability is increased with respect to the $A=0$ case.

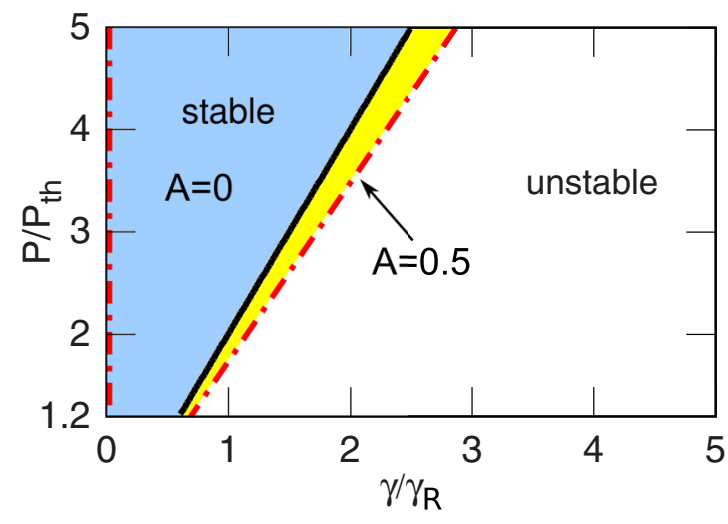

FIG. 3. (Color online) Comparison of stability regions with and without the energy relaxation term included. In both cases, $p=0$. Red dash-dotted lines mark out the stable region for $A=0.5$, which partially overlaps with the stable region for $A=0$. Parameters are $R=1, g=1, g_{R}=2 g, \gamma_{R}=1$, and $\gamma \in[0,5]$.

We note that excitation spectrum of a formally similar hybrid Boltzmann-Gross-Pitaevskii model was calculated recently [39]. In this model, steady states were found to be dynamically (modulationally) stable in the whole parameter range. This stability is a consequence of the assumption of an ideally thermalized reservoir, which translates into an immediate response of the reservoir to the change of the condensate density. This includes the response of the reservoir density distribution $n_{R}(x, t)$. To describe the instability shown in Fig. 1, it is important to take into account that the relatively heavy excitons remain practically immobile on a short-time scale, so that their density distribution can become out of equilibrium (although the reservoir may be thermalized locally).

\section{INHOMOGENEOUS PUMPING}

Experiments with nonresonantly pumped polariton condensates are performed using inhomogeneous, typically Gaussianshaped, pumping beams. In this section, we investigate how the shape of the pump can influence the stability of the condensate.

In Fig. 4, we show examples of dynamics for various parameters of the system with a Gaussian pumping profile, $P(x)=P_{\max } \exp \left(-x^{2} / W^{2}\right)$. The initial state is a small white noise in the polariton field $\psi(x)$. Figures $4(a)$ and $4(b)$ show examples of stable and unstable dynamics, corresponding to Figs. 1(a) and 1(c). There are also a number of other possible nonstationary states, as shown in Figs. 4(c)-4(e), including the oscillating wave packets and breathers. These states, despite the complex dynamics, display correlation length that is comparable with the size of the Gaussian pump. For this reason, we classify these states as "coherent" in contrast to the "incoherent" unstable state of Fig. 4(b). We note that nonstationary condensate states have been previously shown to emerge in two-dimensional models with inhomogeneous pumping $[37,40]$.

In Fig. 5, we show the regions of parameter space where coherent (stationary or nonstationary) states exist. The limits of the regions were determined numerically by solving the GrossPitaevskii equation (2). Clearly, the region of coherence in the 

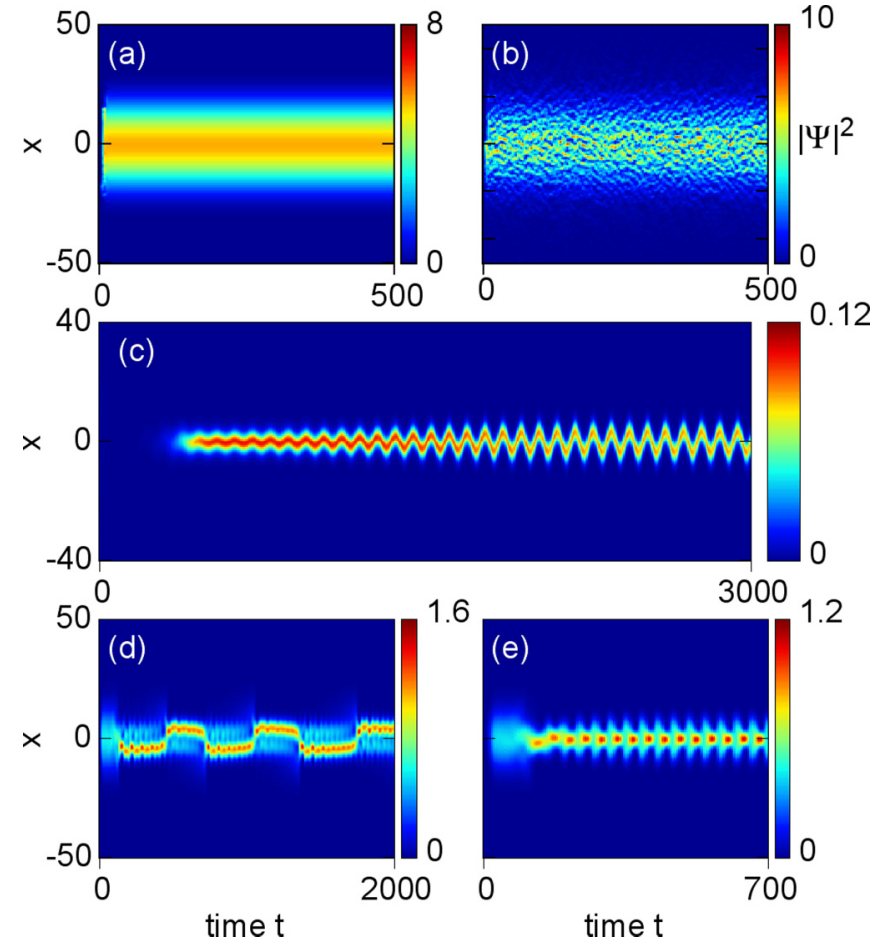

FIG. 4. (Color online) Evolution of the polariton density for Gaussian pumping $P(x)=P_{\max } \exp \left(-x^{2} / W^{2}\right)(A=0)$. (a),(b) The condensate densities in the coherent and incoherent cases, respectively. Parameters are $R=1, \gamma / \gamma_{R}=1.5,5$ for (a) and (b), respectively, $g=0.38, P_{\max } / P_{\text {th }}=1.7, W=44.7$. Frames below show examples of coherent nonstationary states: (c),(d) oscillating wave packets with parameters $R=2.5, \gamma / \gamma_{R}=0.15, g=3$, and $R=$ $1, \gamma / \gamma_{R}=0.7, g=0.38, P_{\max } / P_{\text {th }}=1.8, W=15.8$, respectively, and (e) a breather with parameters $R=1, \gamma / \gamma_{R}=0.7, g=0.38$, $P_{\max } / P_{\text {th }}=1.6, W=15.8$.

case of inhomogeneous pumping is much larger than in the homogeneous case of Fig. 2. Moreover, it expands significantly as the pump size is decreased. The reduction of the pumping spot appears to be a very effective way to stabilize condensates even at high ratios of $\gamma / \gamma_{R}$. Condensates with $\gamma / \gamma_{R}=3.5$ are fully coherent at all pumping powers already at a relatively wide pumping spot with $W=15.8$. We note that the threshold value of $P_{\max }$ for the appearance of the condensate in the case of inhomogeneous pumping is higher than $P_{\text {th }}$ [41]. However, since our pumping profiles are relatively wide, this difference cannot be seen in the area covered by Fig. 5 which begins at $P / P_{\mathrm{th}}=1.2$.

\section{CONCLUSIONS}

In conclusion, we investigated the dynamical stability and its relation to the spatial coherence properties of onedimensional exciton-polariton condensates under nonresonant

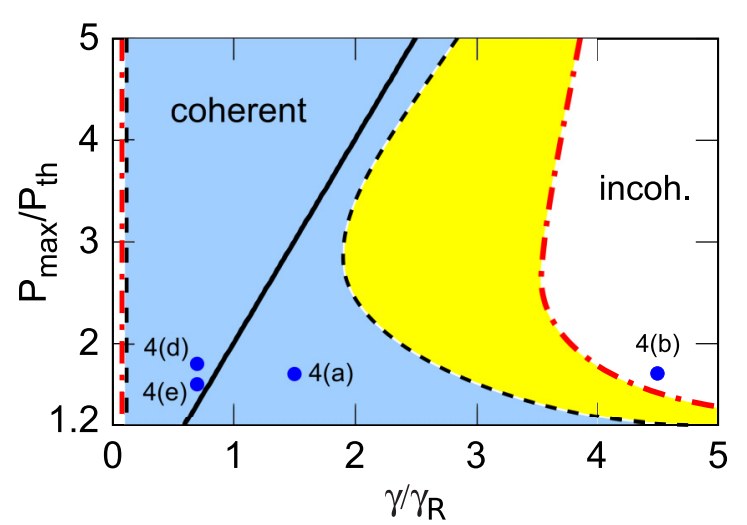

FIG. 5. (Color online) Stability regions for Gaussian pumping profiles. The lines mark out the coherent regions for narrower pumping of width $W=15.8$ (dash-dotted lines) and for wider pumping of width $W=44.7$ (dashed lines). The black solid line corresponds to the analytical stability condition of a homogeneous condensate [19]. Blue points correspond to particular cases from Fig. 4. Parameters are $R=1, g=0.38, g_{R}=2 g$, and $\gamma_{R}=1$. For the choice of scaling parameters as in Fig. 1, the width of the pumping is $W=54 \mu \mathrm{m}$ (dash-dotted lines) and $W=152 \mu \mathrm{m}$ (dashed lines).

pumping. In the case of spatially homogeneous pumping, we found that the instability of the steady state leads to a significant reduction of the condensate coherence. This instability is predicted to occur in the physically relevant case when the loss rate of the exciton reservoir is lower than the loss rate of the exciton polaritons. Since the experiments with inhomogeneous optical pumping reported large coherence lengths [3,14,16-18], we considered two effects that can potentially lead to the stabilization of condensates. These are the effect of polariton energy relaxation and the inhomogeneous pumping profiles. We found that while the former has little effect on the stability, the latter is very effective in stabilizing the condensate, which results in a large coherence length.

It is noteworthy that in the two-dimensional experiment with a top-hat-shaped pumping profile, which is the closest to the homogeneous profile, strong internal dynamics and vortex creation were observed [22], which evidences the existence of a nonstationary state. However, we believe that further experiments, especially with almost-homogeneous or ring-shaped pumps, are necessary to determine the stability limits in the parameter space. Such experiments would also provide verification of the open-dissipative Gross-Pitaevskii model (1) and its modifications widely used in the literature, and allow for the determination of their phenomenological parameters.

\section{ACKNOWLEDGMENTS}

N.B. and M.M. acknowledge support from the National Science Center Grant No. DEC-2011/01/D/ST3/00482. E.A.O. acknowledges support by the Australian Research Council (ARC).
[1] A. Kavokin, J. J. Baumberg, G. Malpuech, and F. P. Laussy, Microcavities (Oxford University Press, Oxford, 2007).
[2] C. Weisbuch, M. Nishioka, A. Ishikawa, and Y. Arakawa, Phys. Rev. Lett. 69, 3314 (1992). 
[3] J. Kasprzak, M. Richard, S. Kundermann, A. Baas, P. Jeambrun, J. M. J. Keeling, F. M. Marchetti, M. H. Szymańska, R. André, J. L. Staehli et al., Nature (London) 443, 409 (2006).

[4] S. Christopoulos, G. B. H. von Högersthal, A. J. D. Grundy, P. G. Lagoudakis, A. V. Kavokin, J. J. Baumberg, G. Christmann, R. Butté, E. Feltin, J.-F. Carlin et al., Phys. Rev. Lett. 98, 126405 (2007).

[5] S. Kéna-Cohen and S. R. Forrest, Nat. Photon. 4, 371 (2010).

[6] J. D. Plumhof, T. Stöferle, L. Mai, U. Scherf, and R. F. Mahrt, Nat. Mater. 13, 247 (2014).

[7] A. Amo, T. C. H. Liew, C. Adrados, R. Houdré, E. Giacobino, A. V. Kavokin, and A. Bramati, Nat. Photon. 4, 361 (2010).

[8] T. Gao, P. S. Eldridge, T. C. H. Liew, S. I. Tsintzos, G. Stavrinidis, G. Deligeorgis, Z. Hatzopoulos, and P. G. Savvidis, Phys. Rev. B 85, 235102 (2012).

[9] D. Ballarini, M. D. Giorgi, E. Cancellieri, R. Houdré, E. Giacobino, R. Cingolani, A. Bramati, G. Gigli, and D. Sanvitto, Nat. Commun. 4, 1778 (2013).

[10] C. Schneider, A. Rahimi-Iman, N. Kim, J. Fischer, I. Savenko, M. Amthor, M. Lermer, A. Wolf, L. Worschech, V. Kulakovskii et al., Nature (London) 497, 348 (2013).

[11] P. Bhattacharya, T. Frost, S. Deshpande, M. Z. Baten, A. Hazari, and A. Das, Phys. Rev. Lett. 112, 236802 (2014).

[12] A. Amo, J. Lefrére, S. Pigeon, C. Adrados, C. Ciuti, I. Carusotto, R. Houdré, E. Giacobino, and A. Bramati, Nat. Phys. 5, 805 (2009).

[13] I. Carusotto and C. Ciuti, Rev. Mod. Phys. 85, 299 (2013).

[14] H. Deng, H. Haug, and Y. Yamamoto, Rev. Mod. Phys. 82, 1489 (2010).

[15] M. Wouters and I. Carusotto, Phys. Rev. Lett. 99, 140402 (2007).

[16] H. Deng, G. S. Solomon, R. Hey, K. H. Ploog, and Y. Yamamoto, Phys. Rev. Lett. 99, 126403 (2007).

[17] E. Wertz, L. Ferrier, D. D. Solnyshkov, R. Johne, D. Sanvitto, A. Lemaître, I. Sagnes, R. Grousson, A. V. Kavokin, P. Senellart et al., Nat. Phys. 6, 860 (2010).

[18] J. Schmutzler, T. Kazimierczuk, O. Bayraktar, M. Aßmann, M. Bayer, S. Brodbeck, M. Kamp, C. Schneider, and S. Höfling, Phys. Rev. B 89, 115119 (2014).

[19] L. A. Smirnov, D. A. Smirnova, E. A. Ostrovskaya, and Y. S. Kivshar, Phys. Rev. B 89, 235310 (2014).

[20] E. Wertz, A. Amo, D. D. Solnyshkov, L. Ferrier, T. C. H. Liew, D. Sanvitto, P. Senellart, I. Sagnes, A. Lemaître, A. V. Kavokin et al., Phys. Rev. Lett. 109, 216404 (2012).
[21] K. G. Lagoudakis, F. Manni, B. Pietka, M. Wouters, T. C. H. Liew, V. Savona, A. V. Kavokin, R. André, and B. DeveaudPlédran, Phys. Rev. Lett. 106, 115301 (2011).

[22] G. Roumpos, M. D. Fraser, A. Löffler, S. Höfling, A. Forchel, and Y. Yamamoto, Nat. Phys. 7, 129 (2010).

[23] J. Szczytko, L. Kappei, J. Berney, F. Morier-Genoud, M. T. Portella-Oberli, and B. Deveaud, Phys. Rev. Lett. 93, 137401 (2004).

[24] D. Bajoni, M. Perrin, P. Senellart, A. Lemaître, B. Sermage, and J. Bloch, Phys. Rev. B 73, 205344 (2006).

[25] Y. Xue and M. Matuszewski, Phys. Rev. Lett. 112, 216401 (2014).

[26] D. S. Petrov, G. V. Shlyapnikov, and J. T. M. Walraven, Phys. Rev. Lett. 85, 3745 (2000).

[27] V. Bagnato and D. Kleppner, Phys. Rev. A 44, 7439 (1991).

[28] I. Bouchoule, K. V. Kheruntsyan, and G. V. Shlyapnikov, Phys. Rev. A 75, 031606 (2007).

[29] M. Steger, G. Liu, B. Nelsen, C. Gautham, D. W. Snoke, R. Balili, L. Pfeiffer, and K. West, Phys. Rev. B 88, 235314 (2013).

[30] C. Ciuti, G. Bastard, and I. Carusotto, Phys. Rev. B 72, 115303 (2005).

[31] M. Wouters and V. Savona, Phys. Rev. B 79, 165302 (2009).

[32] M. Matuszewski and E. Witkowska, Phys. Rev. B 89, 155318 (2014).

[33] A. Chiocchetta and I. Carusotto, Europhys. Lett. 102, 67007 (2013).

[34] V. N. Gladilin, K. Ji, and M. Wouters, Phys. Rev. A 90, 023615 (2014).

[35] M. Wouters and I. Carusotto, Phys. Rev. Lett. 105, 020602 (2010).

[36] G. Li, M. D. Fraser, A. Yakimenko, and E. A. Ostrovskaya, arXiv:1408.1568.

[37] J. Keeling and N. G. Berloff, Phys. Rev. Lett. 100, 250401 (2008).

[38] D. Tanese, H. Flayac, D. Solnyshkov, A. Amo, A. Lemaître, E. Galopin, R. Braive, P. Senellart, I. Sagnes, G. Malpuech et al., Nat. Commun. 4, 1749 (2013).

[39] D. D. Solnyshkov, H. Tercas, K. Dini, and G. Malpuech, Phys. Rev. A 89, 033626 (2014).

[40] M. O. Borgh, J. Keeling, and N. G. Berloff, Phys. Rev. B 81, 235302 (2010).

[41] E. A. Ostrovskaya, J. Abdullaev, A. S. Desyatnikov, M. D. Fraser, and Y. S. Kivshar, Phys. Rev. A 86, 013636 (2012). 